\title{
Effects of Conduction Variation on MHD Natural Convection Flow along a Vertical Flat Plate with Thermal Conductivity
}

\author{
A. K. M. S. Islam ${ }^{\text {1* }}$ M. A. Alim², M. R. Karim ${ }^{3}$ and A. T. M. M. Rahman \\ ${ }^{1}$ Department of Mathematics, Gowripur Govt. College, Mymensingh, Bangladesh \\ ${ }^{2}$ Department of Mathematics, Bangladesh University of Engineering and Technology, \\ Dhaka-1000, Bangladesh \\ ${ }^{3}$ Department of Mathematics, Jagannath University, Dhaka-1100, Bangladesh \\ ${ }^{4}$ Department of Computer Science and Engineering, Dhaka International University, Banani, \\ Dhaka-1213, Bangladesh.
}

Received 23 October 2015, accepted in final revised form 27 June 2016

\begin{abstract}
This paper reports the effect of conduction variation of free convection flow along a vertical flat plate on magnetohydrodynamic (MHD) with thermal conductivity. The governing equations with associated boundary conditions reduce to local non-similarity boundary layer equations for this phenomenon are converted to dimensionless forms using a suitable transformation. The transformed non-linear equations are then solved using the implicit finite difference method together with Keller-box technique. Numerical results of the velocity and temperature profiles, skin friction and surface temperature profiles for different values of the magnetic parameter, the thermal conductivity variation parameter, the Prandtl number and the conduction variation parameters are presented graphically. Detailed discussion is given for the effect of the aforementioned parameters. Opposite scenario is found in skin friction and surface temperature for the thermal conductivity variation parameter. Significant effect is found in skin friction and surface temperature for conduction variation parameter.
\end{abstract}

Keywords: Conductivity; Electrically conducting fluid; Natural convection; Conduction variation.

(C) 2016 JSR Publications. ISSN: 2070-0237 (Print); 2070-0245 (Online). All rights reserved. doi: http://dx.doi.org/10.3329/jsr.v8i3.25459

J. Sci. Res. 8 (3), 237-248 (2016)

\section{Introduction}

Electrically conducting fluid flow in presence of the effect of temperature dependent thermal conductivity flow and heat conduction problems are important from the

* Corresponding author: safiqis@yahoo.com 
technical point of view and such types of problems have received much attention by many researchers.

Model studies of the free convection flows have earned reputations because of their applications in geophysical, geothermal and nuclear engineering problems. Miyamoto et al. [1] studied the effect of axial heat conduction in a vertical flat plate on free convection heat transfer. Pozzi and Lupo [2] investigated the coupling of conduction with laminar convection along a flat plate. Mamun [3] studied the effects of conduction and convection on magnetohydrodynamic (MHD) flow with and without viscous dissipation from a vertical flat plate. Hossain [4] analyzed the viscous and Joule heating effects on MHD free convection flow with variable plate temperature. Rahman et al. [5] investigated the effects of temperature dependent thermal conductivity on MHD free convection flow along a vertical flat plate with heat conduction. Nasrin and Alim [6] studied the combined effects of viscous dissipation and temperature dependent thermal conductivity on MHD free convection flow with conduction and joule heating along a vertical flat plate. Alim et al. [7] investigated Joule heating effect on the coupling of conduction with MHD free convection flow from a vertical flat plate. Chowdhury and Islam [12] analyzed MHD free convection flow of visco-elastic fluid past an infinite porous plate. Alam et al. [13] studied viscous dissipation effects on MHD natural convection flow over a sphere in the presence of heat generation. Islam et al. [14] investigated effects of conduction variation on natural convection flow along a vertical flat plate. Islam et al. [15] studied the Eeffects of thermal conductivity of fluid on free convection flow along a vertical flat plate with transverse conduction. Islam et al. [16] analyzed the effects of conduction variation on MHD natural convection flow along a vertical flat plate.

The present study is to incorporate the idea of the effects of conduction variation on MHD natural convection boundary layer flow along a vertical flat plate with thermal conductivity.

\section{Mathematical Formulation of the Problem}

At first we consider a steady two-dimensional laminar natural convection flow of an electrically conducting, viscous and incompressible fluid along a vertical flat plate of length $l$ and thickness $b$ (Fig. 1). It is assumed that the temperature at the outer surface of the plate is maintained at a constant temperature $T_{b}$, where $T_{b}>T_{\infty}$, the ambient temperature of the fluid. In this work $\bar{y}$-axis i.e. normal direction to the surface and $\bar{x}$-axis is taken along the flat plate. The coordinate system and the configuration are shown in Fig. 1. 


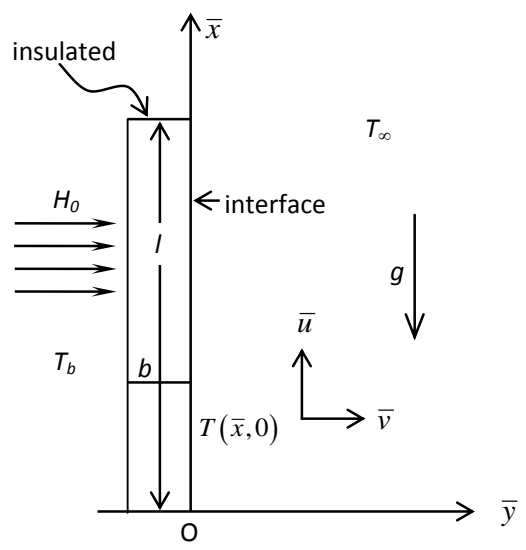

Fig. 1. Physical model and coordinate system.

The governing equations of such laminar flow with viscous dissipation and also thermal conductivity variation along a vertical flat plate under the Boussinesq approximations $\rho=\rho_{\infty}\left[1-\beta\left(T_{b}-T_{\infty}\right)\right]$, where $\rho_{\infty}$ and $T_{\infty}$ are the density and temperature respectively outside the boundary layer. For the present problem we see the equations of continuity, momentum and energy are as below

$\frac{\partial \bar{u}}{\partial \bar{x}}+\frac{\partial \bar{v}}{\partial \bar{y}}=0$

$\bar{u} \frac{\partial \bar{u}}{\partial \bar{x}}+\bar{v} \frac{\partial \bar{u}}{\partial \bar{y}}=v \frac{\partial^{2} \bar{u}}{\partial \bar{y}^{2}}+g \beta\left(T_{f}-T_{\infty}\right)-\frac{\sigma H_{0}^{2} \bar{u}}{\rho}$

$\bar{u} \frac{\partial T_{f}}{\partial \bar{x}}+\bar{v} \frac{\partial T_{f}}{\partial \bar{y}}=\frac{1}{\rho C_{p}} \frac{\partial}{\partial \bar{y}}\left(\kappa_{f} \frac{\partial T_{f}}{\partial \bar{y}}\right)$

Here $\beta$ is coefficient of volume expansion. The temperature dependent thermal conductivity, which is used by Rahman [5] as follows $\kappa_{f}=\kappa_{\infty}\left[1+\delta\left(T_{f}-T_{\infty}\right)\right](4)$

Where $\kappa_{\infty}$ is the thermal conductivity of the ambient fluid and $\delta$ is a constant, defined as $\delta=\frac{1}{\kappa_{f}}\left(\frac{\partial \kappa}{\partial T}\right)_{f}$. The appropriate boundary condition to be satisfied by the above equations are

$\left.\begin{array}{l}\bar{u}=0, \bar{v}=0 \\ T_{f}=T(\bar{x}, 0), \frac{\partial T_{f}}{\partial \bar{y}}=\frac{\kappa_{s}}{b \kappa_{f}}\left(T_{f}-T_{b}\right)\end{array}\right\}$ on $\bar{y}=0, \bar{x}>0$

$\bar{u} \rightarrow 0, T_{f} \rightarrow T_{\infty}$ as $\bar{y} \rightarrow \infty, \bar{x}>0$

The non-dimensional governing equations and boundary conditions can be obtained from equations (1) - (3) using the following dimensionless quantities 


$$
\begin{aligned}
& x=\frac{\bar{x}}{l}, \quad y=\frac{\bar{y}}{l} G r^{\frac{1}{4}}, u=\frac{\bar{u} l}{v} G r^{-\frac{1}{2}}, v=\frac{\bar{v} l}{v} G r^{-\frac{1}{4}}, \\
& \theta=\frac{T_{f}-T_{\infty}}{T_{b}-T_{\infty}}, G r=\frac{g \beta l^{3}\left(T_{b}-T_{\infty}\right)}{v^{2}}
\end{aligned}
$$

where $l$ is the length of the plate, $G r$ is the Grashof number, $\theta$ is the dimensionless temperature.

Now from equations (1)-(3), we get using the following dimensionless equations

$$
\begin{aligned}
& \frac{\partial u}{\partial x}+\frac{\partial v}{\partial y}=0 \\
& u \frac{\partial u}{\partial x}+v \frac{\partial u}{\partial y}+M u=\frac{\partial^{2} u}{\partial y^{2}}+\theta \\
& u \frac{\partial \theta}{\partial x}+v \frac{\partial \theta}{\partial y}=\frac{1}{\operatorname{Pr}}(1+\gamma \theta) \frac{\partial^{2} \theta}{\partial y^{2}}+\frac{\gamma}{\operatorname{Pr}}\left(\frac{\partial \theta}{\partial y}\right)^{2}
\end{aligned}
$$

where $\operatorname{Pr}=\frac{\mu C_{p}}{\kappa_{\infty}}$ is the Prandtl number, $\gamma=\delta\left(T_{b}-T_{\infty}\right)$ is the non-dimensional thermal conductivity variation parameter and $M=\frac{\sigma H_{0}^{2} l^{2}}{\mu G r^{1 / 2}}$ is the dimensionless magnetic parameter. The corresponding boundary conditions (5) then take the following form $u=0, v=0, \theta-1=(1+\gamma \theta) P \frac{\partial \theta}{\partial y}$ on $y=0, x>0$

$u \rightarrow 0, \theta \rightarrow 0$ as $y \rightarrow \infty, x>0$ here $P=\left(\frac{\kappa_{\infty} b}{\kappa_{s} l}\right) G r^{\frac{1}{4}}$ is the conduction parameter. The described problem is governed by the coupling parameter $P$.

To solve the equations (8) and (9) subject to the boundary conditions (10) the following transformations are proposed by Merkin and Pop [8]

$$
\begin{aligned}
& \psi=x^{\frac{4}{5}}(1+x)^{-\frac{1}{20}} f(x, \eta) \\
& \eta=y x^{-\frac{1}{5}}(1+x)^{-\frac{1}{20}} \\
& \theta=x^{\frac{1}{5}}(1+x)^{-\frac{1}{5}} h(x, \eta)
\end{aligned}
$$

here $\eta$ is the similarity variable and $\psi$ is the non-dimensional stream function which satisfies the continuity equation and is related to the velocity components in the usual way as

$u=\frac{\partial \psi}{\partial y}$ and $v=-\frac{\partial \psi}{\partial x}$. 
Moreover, $h(x, \eta)$ represents the non-dimensional temperature. The momentum and energy equations are transformed for the new co-ordinate system. At first, the velocity components are expressed in terms of the new variables for this transformation. Now the equations take the following forms

$$
\begin{array}{r}
f^{\prime \prime \prime}+\frac{16+15 x}{20(1+x)} f f^{\prime \prime}-\frac{6+5 x}{10(1+x)} f^{\prime 2}-M x^{\frac{2}{5}}(1+x)^{\frac{1}{10}} f^{\prime}+h=x\left(f^{\prime} \frac{\partial f^{\prime}}{\partial x}-f^{\prime \prime} \frac{\partial f}{\partial x}\right) \\
\frac{1}{\operatorname{Pr}} h^{\prime \prime}+\frac{\gamma}{\operatorname{Pr}}\left(\frac{x}{1+x}\right)^{\frac{1}{5}} h h^{\prime \prime}+\frac{\gamma}{\operatorname{Pr}}\left(\frac{x}{1+x}\right)^{\frac{1}{5}} h^{\prime 2}+\frac{16+15 x}{20(1+x)} f h^{\prime} \\
-\frac{1}{5(1+x)} f^{\prime} h=x\left(f^{\prime} \frac{\partial h}{\partial x}-h^{\prime} \frac{\partial f}{\partial x}\right)
\end{array}
$$

where prime denotes partial differentiation with respect to $\eta$. The boundary conditions as mentioned in equation (10) then take the following form

$$
\begin{aligned}
f(x, 0) & =f^{\prime}(x, 0)=0 \\
h^{\prime}(x, 0) & =\frac{x^{\frac{1}{5}}(1+x)^{-\frac{1}{5}} h(x, 0)-1}{(1+x)^{-\frac{1}{4}}+\gamma x^{\frac{1}{5}}(1+x)^{-\frac{9}{20}} h(x, 0)} \\
f^{\prime}(x, \infty) \rightarrow 0, \quad h(x, \infty) \rightarrow 0 & \rightarrow 0
\end{aligned}
$$

From the process of numerical computation, in practical point of view, it is important to calculate the values of the surface shear stress in terms of the skin friction coefficient. This can be written in the non-dimensional form as Molla et al. [9]

$C_{f}=\frac{G r^{-\frac{3}{4}} l^{2}}{\mu v} \tau_{w}$

where $\tau_{w}\left[=\mu\left(\begin{array}{lll}\partial & \bar{u} / \partial & \bar{y})_{\bar{y}=0}\end{array}\right]\right.$ is the shearing stress. Using the new variables described in (6), the local skin friction coefficient can be written as

$C_{f x}=x^{\frac{2}{5}}(1+x)^{-\frac{3}{20}} f^{\prime \prime}(x, 0)$

In practical point of view, it is important to calculate the values of the surface temperature. The numerical values of the surface temperature are obtained from the relation. This can written in the non-dimensional form as

$\theta(x, 0)=x^{\frac{1}{5}}(1+x)^{-\frac{1}{5}} h(x, 0)$

\section{Numerical Method of Solution}

This paper reports the effect of conduction variation on electrically conducting fluid in natural convection flow for temperature dependent thermal conductivity along a 
vertical flat plate with MHD. The set of equations (12) and (13) together with the boundary conditions (14) are solved by applying implicit finite difference method with Keller-box elimination scheme [10], which is well documented by Cebeci and Bradshaw [11] and widely used by Keller et al. [4].

\section{Results and Discussion}

The main objective of the present study is to analyze the effect of conduction variation due to temperature dependent thermal conductivity on free convective flow along a vertical flat plate in presence of strong magnetic field. In this simulation the values of the Prandtl number $\mathrm{Pr}$ are considered to be $0.733,1.00,1.50,2.00$ and 2.50 that corresponds to hydrogen, steam, water, methyl chloride and sulfur dioxide respectively.

The velocity and the temperature profiles obtained from the solutions of equations (12) and (13) are depicted in Figs. 2-5. Also the local skin friction and the surface temperature obtained from the solutions of equations (16) and (17) are depicted in Figs. 6-9. Numerical computation are carried out for a range of magnetic parameter $M$ $=0.01,0.21,0.41,0.61,0.81$, thermal conductivity variation parameter $\gamma=0.01,0.11$, $0.21,0.31,0.41$ and conduction variation parameter $P=0.30,0.60,0.80,1.00,1.20$.

The effect of magnetic parameter $M$ on the velocity and temperature profiles against $\eta$ within the boundary layer with $\gamma=0.01, P r=0.733$ and $P=0.30$ are shown in Figs. 2(a) and 2(b), respectively. It is seen from Fig. 2(a) that the velocity decreases within the boundary layer with the increasing values of $M$. From Fig. 2(b) it is observed that the temperature increases within the boundary layer with the increasing values of $M$. It means that the velocity boundary layer and the thermal boundary layer thickness expand for large values of $M$.

The effect of thermal conductivity variation parameter $\gamma$ on the velocity and temperature profiles against $\eta$ within the boundary layer with $M=0.01, \operatorname{Pr}=0.733$ and $P=0.30$ are shown in Figs. 3(a) and 3(b), respectively. It is observed that the velocity and the temperature increase within the boundary layer with the increasing values of $\gamma$. It means that the velocity boundary layer and the thermal boundary layer thickness expand for large values of $\gamma$.

Figs. 4(a) and 4(b) illustrate the velocity and temperature profiles against $\eta$ for different values of Prandtl number $\operatorname{Pr}$ with $M=0.01, \gamma=0.01$ and $P=0.30$. From Fig. $4(\mathrm{a})$, it can be observed that the velocity decreases as well as its position moves toward the interface with the increasing Pr. From Fig. 4(b), it is seen that the temperature profiles shift downward with the increasing values of $\mathrm{Pr}$. Velocity and temperature boundary layer both are much more clear and decrease for large Prandtl number.

Figs. 5(a) and 5(b) describe the velocity and temperature profiles against $\eta$ for different values of conduction variation parameter $P$ with $M=0.01, \gamma=0.01$ and $\operatorname{Pr}=$ 0.733. From Fig. 5(a), it can be observed that the velocity decreases as well as its position moves outward the interface with the increasing values of $P$. From Fig. 5(b), it 
is seen that the temperature profiles also the same as increase within the boundary layer. It means that the velocity boundary layer and the thermal boundary layer thickness increase for large values of $P$ from 0.30 to 1.20 . There is an inverse trend visible in velocity profiles.

Figs. 6(a) and 6(b) illustrate the effect of the magnetic parameter on the local skin friction coefficient $C_{f x}$ and surface temperature distribution $\theta(x, 0)$ against $x$ with $\gamma=$ $0.01, P r=0.733$ and $P=0.30$. It is seen from Fig. 6(a) that the skin friction increases monotonically along the downward direction of the plate for a particular value of $M$. It is also seen that the local skin friction coefficient decreases for the increasing values of $M$. From Fig. 6(b), it can be seen that the surface temperature increases due to the increasing value along the positive $x$ direction for a particular $M$. It is also observed that the surface temperature decreases for increasing values of $M$. This means that there is an inverse case exists between skin friction and surface temperature which is remarkable.

Figs. 7(a) and 7(b) illustrate the effect of the thermal conductivity variation parameter on the skin friction coefficient and surface temperature against $x$ with $M=$ $0.01, P r=0.733$ and $P=0.30$. It is seen from Fig. 7(a) that the skin friction increases monotonically along the upward direction of the plate for a particular value of $\gamma$. It is also seen that the local skin friction coefficient increases for the increasing values of $\gamma$. From Fig. 7(b), it can be seen that the surface temperature increases due to the increasing value along the positive $x$ direction for a particular $\gamma$. We observed that both skin friction and surface temperature profiles are opposite.

Figs. 8(a) and 8(b) deal with the effect of Prandtl number $P r$ on the local skin friction coefficient and surface temperature against $x$ with $M=0.01, \gamma=0.01$ and $P=$ 0.30. It can be observed from Fig. 8(a) that the skin friction coefficient increases monotonically along $x$ axis for a particular value of $P r$. It can also be noted that the skin friction coefficient decreases for the increasing values of Pr. From Fig. 8(b), it can be seen that the surface temperature distribution decreases due to the increases along the positive $x$ direction for a particular value of $P r$.

The variation of the skin friction and surface temperature for different values of $P$ with $M=0.01, \gamma=0.01$, and $P r=0.733$ at different positions are illustrated in Figs. 9(a) and 9(b), respectively. In Fig. 9(a) we see that the skin friction decreases for increasing values of $P$. It can also be noted from Fig. 9(a) that the skin friction increases monotonically along $x$ axis for a particular value of $P$. Again Fig. 9(b) shows that the surface temperature decreases for increasing values of $P$. We observe from the profiles of skin friction and surface temperature that they are opposite. 

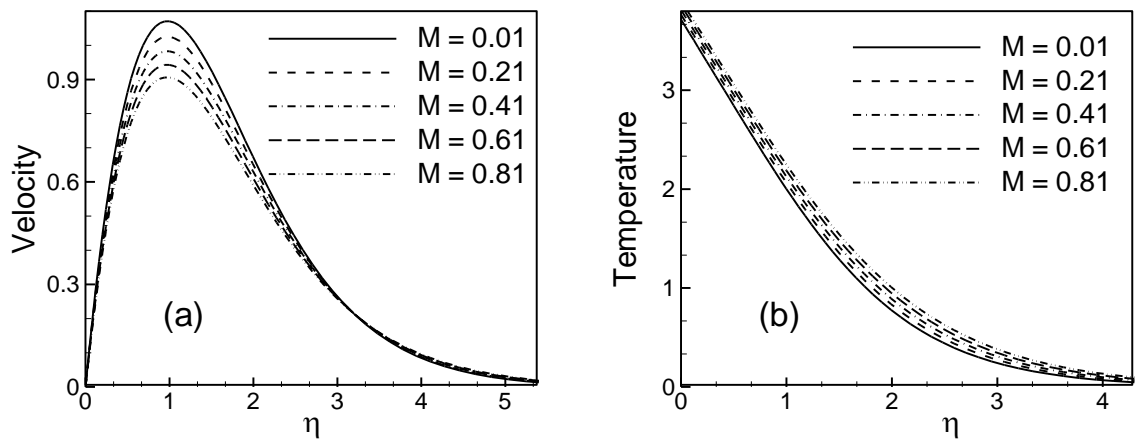

Fig. 2(a). Velocity and (b) temperature profiles against $\eta$ for different values of $M$ with $\gamma=0.01$, $P r=0.733$ and $P=0.30$.
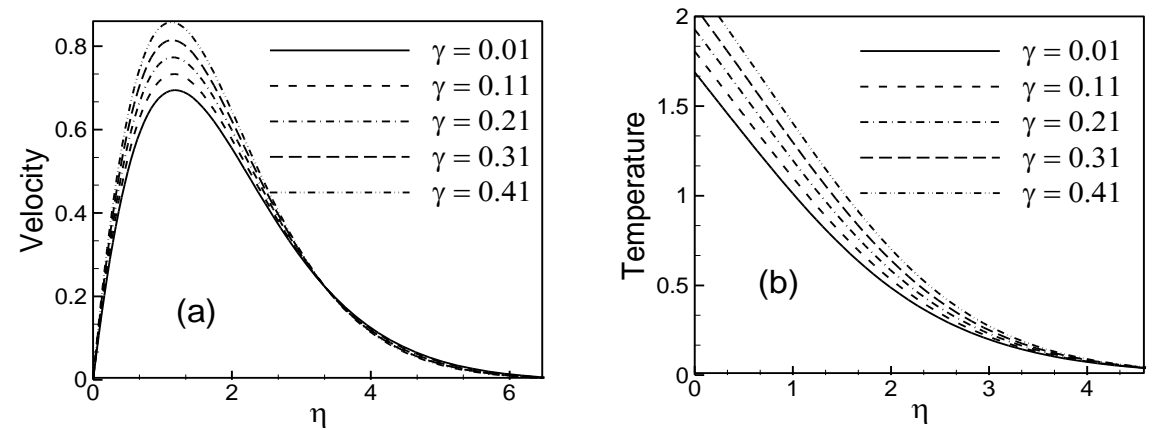

Fig. 3(a). Velocity and (b) temperature profiles against $\eta$ for different values of $\gamma$ with $M=0.01$ $\operatorname{Pr}=0.733$ and $P=0.30$.
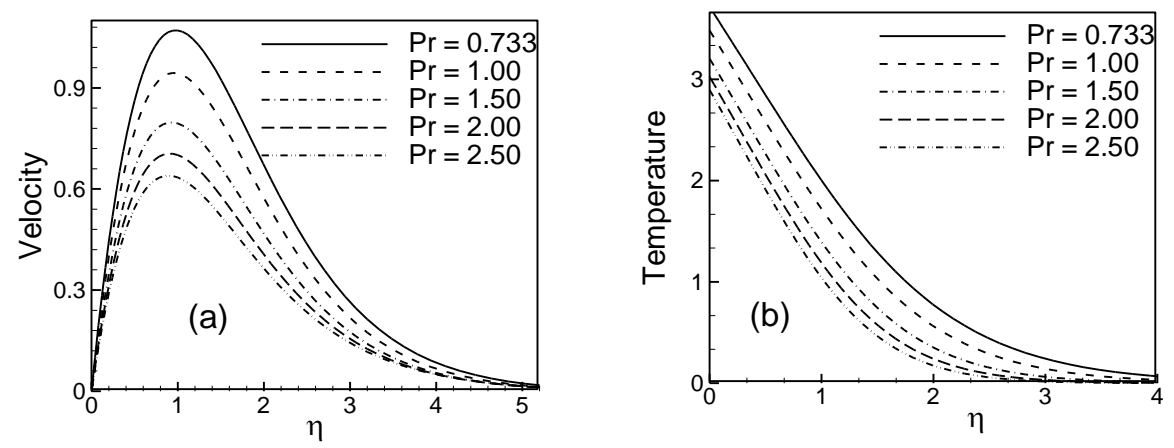

Fig. 4(a). Velocity and (b) temperature profiles against $\eta$ for different values of $\operatorname{Pr}$ with $M=$ $0.01, \gamma=0.01$ and $P=0.30$. 

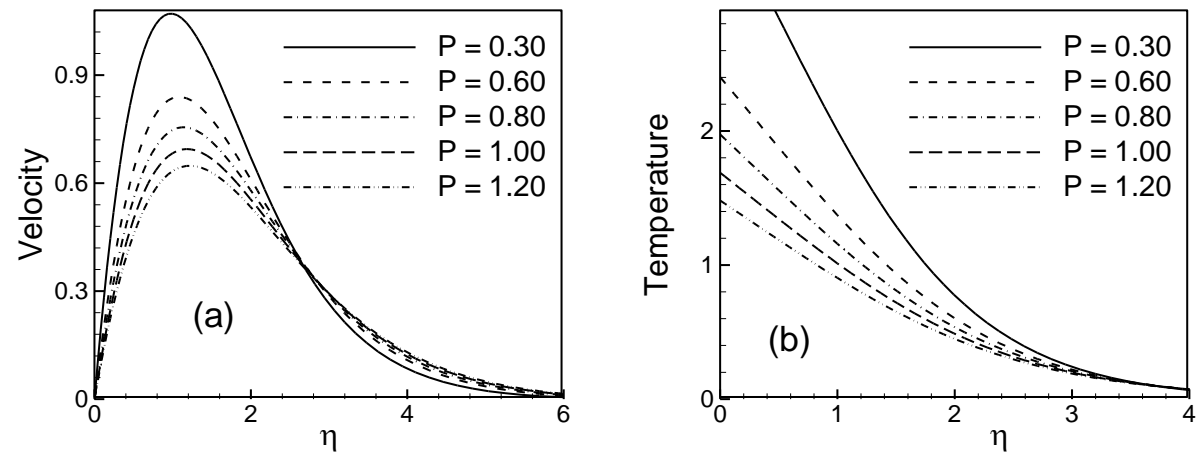

Fig. 5(a). Velocity and (b) temperature profiles against $\eta$ for different values of $P$ with $M=$ $0.01, \gamma=0.01$ and $\operatorname{Pr}=0.733$.
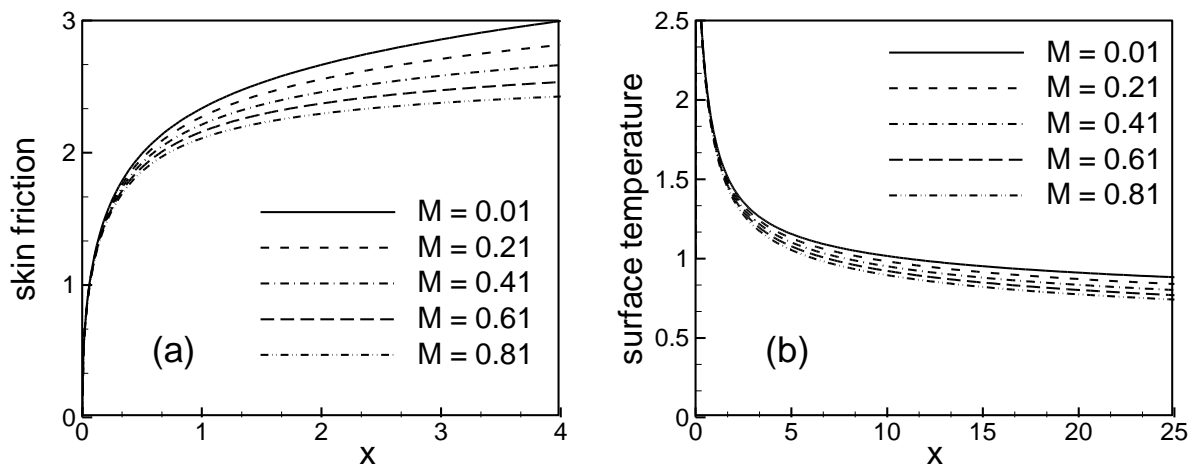

Fig. 6(a). Local skin friction coefficient and (b) surface temperature distribution against $x$ for different values of $M$ with $\gamma=0.01, \operatorname{Pr}=0.733$ and $P=0.30$.
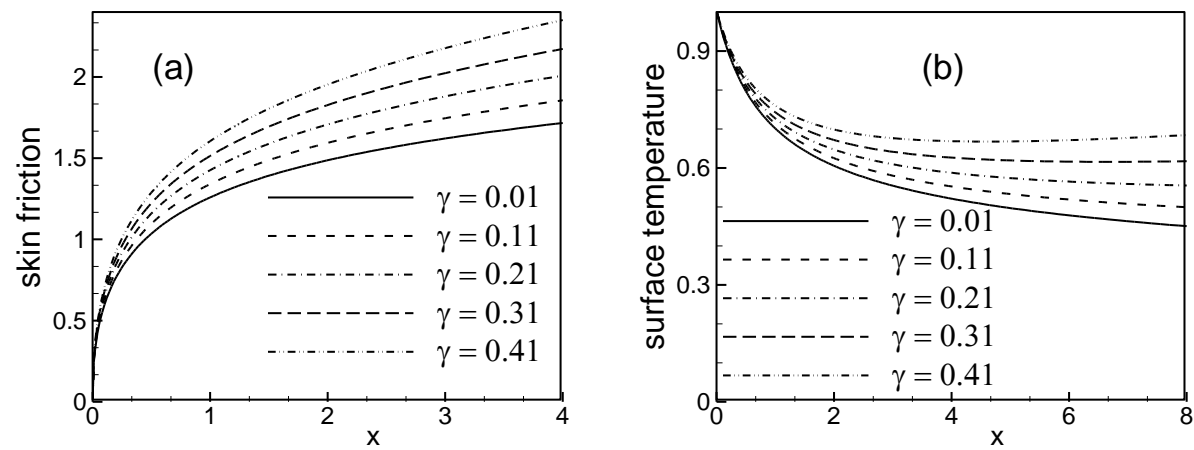

Fig. 7(a). Local skin friction coefficient and (b) surface temperature distribution against $x$ for different values of $\gamma$ with $M=0.01, \operatorname{Pr}=0.733$ and $P=0.30$. 

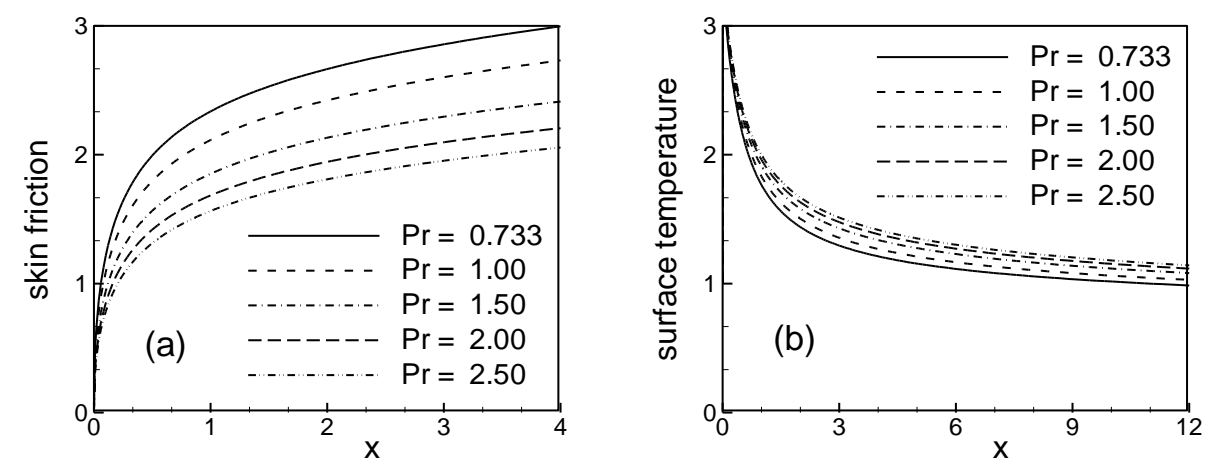

Fig. 8(a). Local skin friction and (b) surface temperature against $x$ for different values of $\operatorname{Pr}$ with $M=0.01, \gamma=0.01$ and $P=0.30$.
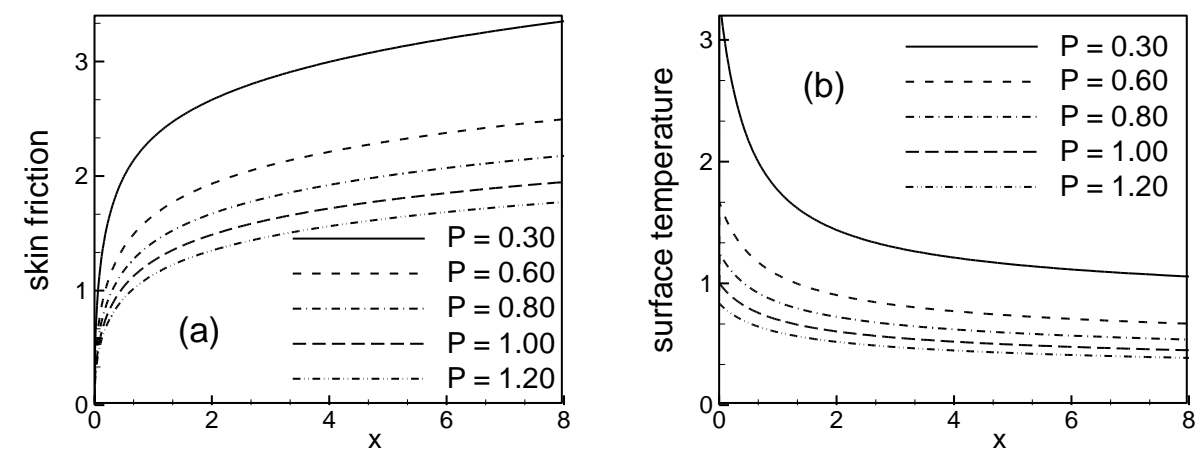

Fig. 9(a). Local skin friction and (b) surface temperature against $x$ for different values of $P$ with $M=0.01, \gamma=0.01$ and $\operatorname{Pr}=0.733$.

\section{Conclusion}

The effects of conduction variation on MHD free convection boundary layer flow along a vertical flat plate with temperature dependent thermal conductivity have been studied in this paper. From the present investigation the following conclusions may be drawn

The velocity within the boundary layer increases for decreasing values of $M, P r, P$ and for increasing values of $\gamma$. There is an inverse trend is found in velocity profile for $P$ which is remarkable.

The temperature within the boundary layer increases for increasing values of $M, \gamma$ and decreases for increasing values of $\mathrm{Pr}, \mathrm{P}$.

The local skin friction coefficient decreases for the increasing values of $M, \operatorname{Pr}, P$ and increases for increasing values of $\gamma$.

An increase in the values of $\gamma$ and $\operatorname{Pr}$ leads to an increase in surface temperature.

On the other hand, this decreases for increasing values of $M, P$. 


\begin{tabular}{|c|c|c|}
\hline & \multirow[b]{2}{*}{ Greek Symbols } \\
\hline \multicolumn{2}{|c|}{ Nomenclature } & \\
\hline$C_{f x}$ & Local skin friction coefficient & \\
\hline$C_{\mathrm{p}}$ & Specific heat at constant pressure & $\beta$ Co-efficient of thermal \\
\hline$F$ & Dimensionless stream function & expansion \\
\hline$g$ & Acceleration due to gravity & $\gamma \quad$ Thermal conductivity variation \\
\hline$G r$ & Grashof number & parameter \\
\hline$h$ & Dimensionless temperature & $\nabla \quad$ Vector differential operator \\
\hline$l$ & Length of the plate & $\eta \quad$ Similarity variable \\
\hline$M$ & Magnetic parameter & $\kappa_{\infty}$ Thermal conductivity of the \\
\hline$P$ & Conduction variation parameter & ambient fluid \\
\hline & Prandtl number & $\kappa$ Thermal conductivity of the \\
\hline & Temperature of the interface & 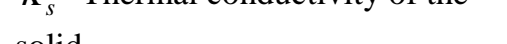 \\
\hline $\begin{array}{l}T_{b} \\
\text { plate }\end{array}$ & Temperature at outside surface of the & $\begin{array}{l}\text { solid } \\
\kappa_{f} \text { Thermal conductivity of the }\end{array}$ \\
\hline$T_{f}$ & Temperature of the fluid & f \\
\hline$T_{\infty}$ & Temperature of the ambient fluid & $\mu \quad$ Viscosity of the fluid \\
\hline$\underline{\bar{u}}$ & Velocity component in $\mathrm{x}$ - direction & $\mu_{e} \quad$ Magnetic permeability of the \\
\hline & Velocity component in y- direction & fluid \\
\hline $\begin{array}{l}u \\
\mathrm{x}-\operatorname{dir}\end{array}$ & $\begin{array}{l}\text { Dimensionless velocity component in } \\
\text { ction }\end{array}$ & 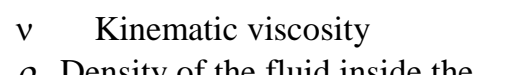 \\
\hline $\begin{array}{l}v \\
y-\operatorname{dir}\end{array}$ & $\begin{array}{l}\text { Dimensionless velocity component in } \\
\text { ction }\end{array}$ & $\begin{array}{l}\rho \text { Density of the fluid inside the } \\
\text { boundary layer }\end{array}$ \\
\hline $\bar{x}$ & Cartesian co-ordinates & $\sigma$ Electrical conductivity of the \\
\hline $\bar{y}$ & Cartesian co-ordinates & $\tau_{w} \quad$ Shearing stress \\
\hline$x$ & Dimensionless Cartesian co-ordinates & $\Psi \quad$ Stream function \\
\hline$y$ & Dimensionless Cartesian co-ordinates & \\
\hline
\end{tabular}

\section{References}

1. M. Miyamoto, J. Sumikawa, T. Akiyoshi, and T. Nakamura, Int. J. Heat Mass Transfer 23(11), 1545 (1980). http://dx.doi.org/10.1016/0017-9310(80)90158-1

2. A. Pozzi and M. Lupo, Int. J. Heat Mass Transfer 31(9), 1807 (1988). http://dx.doi.org/10.1016/0017-9310(88)90195-0

3. A. A. Mamun, M. Phil thesis, Department of Mathematics, BUET, Bangladesh (2005).

4. M. A. Hossain, Int. J. Heat transfer 35(12), 3485 (1992). http://dx.doi.org/10.1016/00179310(92)90234-J

5. M. M. Rahman, A. A. Mamun, M. A. Azim, and M. A. Alim, Nonlinear Analysis: Modeling Control 13(4), 513 (2008).

6. R. Nasrin and M. A. Alim, J. Naval Arch. Marine Eng. JNAME 6(1), 30 (2009).

7. M. A. Alim, M. Alam, and A. A. Mamun, Nonlinear Analysis: Modeling Control 12(3), 307 (2007).

8. J. H. Merkin, and I. Pop, Int. J. Heat Mass Transfer 39, 1527 (1996). http://dx.doi.org/10.1016/0017-9310(95)00238-3

9. M. Molla, M. A. M, Rahman, and T. L. Rahman, J. Arch. Marine Eng. 2, 53 (2005).

10. H. B. Keller, Annual Rev. Fluid Mech. 10, 417 (1978). http://dx.doi.org/10.1146/annurev.fl.10.010178.002221

11. T. Cebeci and P. Bradshaw, Physical and Computational Aspects of Convective Heat Transfer (Springer, New York, 1984). http://dx.doi.org/10.1007/978-3-662-02411-9 
12. M. K. Chowdhury and M. N. Islam, Int. J. Heat and Mass Transfer 36(5), 439 (2000). http://dx.doi.org/10.1007/s002310000103

13. M. M. Alam, M. A. Alim, and M. M. K. Chowdhury, Nonlinear Analysis: Modeling Control 12(4), 447 (2007).

14. A. K. M. S. Islam, M. A. Alim, M. R. Karim, and A. T. M. M. Rahman, Bangladesh J. Phys. 15, 83 (2014). http://dx.doi.org/10.4135/9781483346342.n27

15. A. K. M. S. Islam, M. A. Alim, M. R. Karim, and A. T. M. M. Rahman, J. Mech. Eng. 44(2), 105 (2014).

16. A. K. M. S. Islam, M. A. Alim, M. R. Karim, and A. T. M. M. Rahman, J. Bangladesh Math. Soc. 34, 63 (2014). 